\title{
HEAT CONDUCTION IN MOVING SOLIDS USING GREEN'S FUNCTION
}

\author{
S. S. Ribeiro ${ }^{a}$, \\ G. Guimarães ${ }^{b}$, \\ A. Fernandes ${ }^{a}$, \\ and G. C. Oliveira ${ }^{a}$ \\ ${ }^{\mathrm{a}}$ Universidade Federal de Uberlândia \\ Departamento de Engenharia Mecânica \\ Bairro Santa Mônica \\ CEP 38408-100, Uberlândia, MG, Brasil \\ sidney.ribeiros@hotmail.com

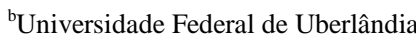 \\ Departamento de Engenharia Mecânica \\ Bairro Santa Mônica \\ CEP 38408-100, Uberlândia, MG, Brasil \\ Received: April 30, 2015 \\ Revised: Mai 13, 2015 \\ Accepted: Mai 16, 2015 \\ $\mathrm{x} \quad$ cartesian coordinate, $\mathrm{m}$ \\ $\mathrm{c}_{\mathrm{p}} \quad$ constant pressure specific heat, $\mathrm{J} /(\mathrm{KgK})$ \\ $\mathrm{k}$ thermal diffusivity, $\mathrm{W} /(\mathrm{mK})$ \\ W auxiliary variable, ${ }^{\circ} \mathrm{C}$ \\ $\mathrm{P} \quad$ fixed point of heat source, $\mathrm{m}$
}

ABSTRACT

Thermal problems involving moving heat sources occur in various engineering applications, such as welding processes, heat treatment furnaces and other treatments. Typically, in these cases, the precise formulation of the numerical solution due to the high complexity of the heat diffusion governing equation, boundary condition, including convection terms. This work proposes a mathematical analysis, analytical solution, verification using other solutions and comparison with numerical solution of a 1D transient thermal model based on Green'n functions, considering a solid moving at a constant speed along a cartesian coordinated.

Keywords: heat transfer, green's function, moving solids

\section{NOMENCLATURE}

$\mathrm{L} \quad$ length, $\mathrm{m}$

G Green function

t time, $\mathrm{s}$

T temperature, ${ }^{\circ} \mathrm{C}$

$\mathrm{V}$ velocity, $\mathrm{m} / \mathrm{s}$

\section{Greek symbols}

$\begin{array}{ll}\alpha & \text { thermal diffusivity, } \mathrm{m}^{2} / \mathrm{s} \\ \xi & \text { auxiliary cartesian coordinate, } \mathrm{m} \\ \delta & \text { Dirac delta function } \\ \rho & \text { specific mass, } \mathrm{Kg} / \mathrm{m}^{3} \\ \tau & \text { response of heat pulse } \\ \mathrm{B} & \text { eigenvalue }\end{array}$

\section{INTRODUCTION}

The analytical solutions represent an important tool for solving engineering problems, since they can be used for validation approximate solutions facilitate the analysis and understanding of physical problems Fernandes (2009), to provide information enabling accurate information about the behavior of the distribution of temperature and heat flux that can be difficult to see from the numerical solutions. However, there is a bigger difficulty mathematics in obtaining analytical solutions involving interpretation and calculation of a thermal problem, as suggested (Fernandes 2009): The complexity of a thermal model, from the point of view to obtain analytical solutions typically found in multi-dimensional problems transient submitted to in homogeneities as prescribed flow boundary conditions, heat generation or boundary conditions as temperature varying with time.

Several physical situations or processes can be analyzed from obtaining the temperature field in a solid exposed to various forms of heating, fixed or moving. For example a moving laser heating of solid surface Araya and Gutierrez (2006), friction welding process Mohammad et al. (2013) and even a computational performance analysis involving obtaining thermo physical properties in inverse problems in heat conduction Fernandes et al (2010).

The motivation for this work is due to the few existing analytical solutions with sources moving in the literature, given the mathematical complexity, analytical solutions are usually obtained with simplified and restrictive assumptions. This paper presents a mathematical analysis and methodology for obtaining an analytical solution 1D transient problem in a moving solid heated by a heat source.

The relevance of this work is due to the few 
existing analytical solutions to moving sources in in the literature, given the mathematical complexity, analytical solutions are normally obtained with simplified hypotheses and restrictive. This paper presents a mathematical analysis and methodology for obtaining analytical solutions in multidimensional, transient problems involving a moving solid heated by a heat source, which can be applied in several engineering areas.

\section{THEORY}

The mathematical model that describes 1D heat conduction in a heated solid by a moving source is:

$$
\frac{\partial^{2} T}{\partial \mathrm{X}^{2}}+\frac{1}{k} g(x, t)=\frac{1}{\alpha}\left(\frac{\partial T}{\partial t}+v \frac{\partial T}{\partial \mathrm{X}}\right)
$$

where $\mathrm{V}$ is the solid speed in the positive direction through a fixed volume control, the boundary conditions of the first kind are given by:

$$
\begin{aligned}
& \mathrm{T}(0, \mathrm{t})=\mathrm{T}_{x 1} \\
& \mathrm{~T}(\mathrm{~L}, \mathrm{t})=\mathrm{T}_{x 2}
\end{aligned}
$$

and boundary conditions of second and third kinds are given by:

$$
\begin{aligned}
& -k \frac{d T}{d x}=\mathrm{h}_{x 1}\left[T_{\infty}-T(0, t)\right]+q_{x 1} \\
& k \frac{d T}{d x}=\mathrm{h} 2\left[T(L, t)-T_{\infty}\right]+q_{x 2}
\end{aligned}
$$

and initial condition:

$$
\mathrm{T}(\mathrm{x}, 0)=\mathrm{F}(\mathrm{x})
$$

For the $1 \mathrm{D}$ case with heat flux at $\mathrm{x}=0$ a variable chance is necessary because of difficult in geometric interpretation, so, consider a constant internal heat generation in any position between 0 and $\mathrm{L}$, and spatial variation we consider a Dirac delta function, so, we have:

$$
\mathrm{g}(\mathrm{x}, \mathrm{t})=\mathrm{g}(\mathrm{x}, \mathrm{t}) \delta(\mathrm{x}-\mathrm{vt})
$$

Two interpretations can be considered, the solid move and the heat source is fixed or the solid if fixed and heat source is move, we consider the second case, the heat source is fixed in any position between 0 or $\mathrm{L}$, while the solid move along positive $\mathrm{x}$ axis.

For this interpretation we consider a new variable Özisik (1993):

$$
\xi=x-v t
$$

Next figures illustrates this two cases:

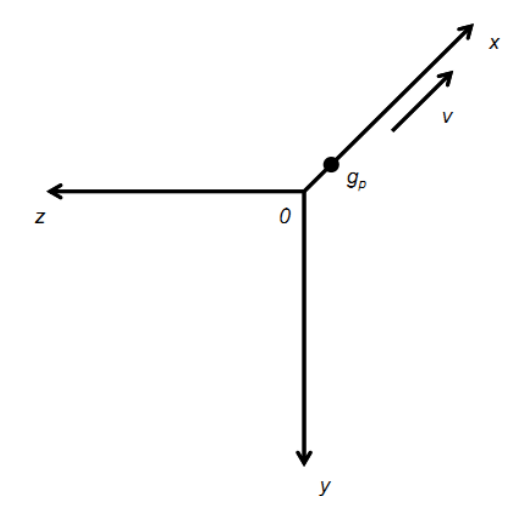

Figure 1. Heat source moving with $\mathrm{x}$ axis.

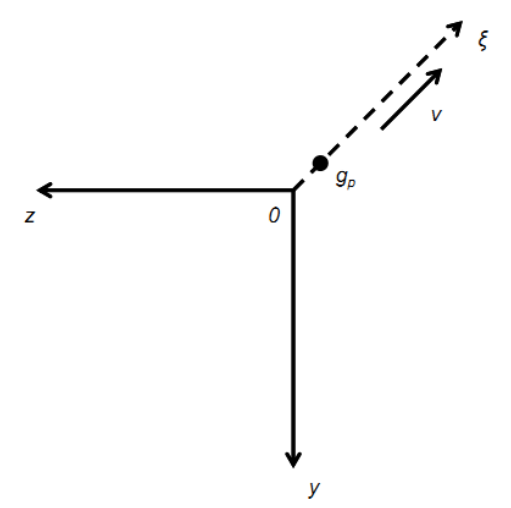

Figure 2. Heat source is fixed while $\mathrm{x}$ axis move. form:

Thus, the governing equation, Eq. (1), take the

$\frac{\partial^{2} T}{\partial \xi^{2}}+\frac{1}{k} g(\xi, t) \delta\left(\xi-P_{\xi}\right)=\frac{1}{\alpha}\left(\frac{\partial T}{\partial t}+v \frac{\partial T}{\partial \xi}\right)$

Similarly the boundary condition and initial condition are described for equations 2-4, but with new variable.

Notice that Eq. (7) is dependent the velocity term, for the application Green function method we have eliminated this term. For this we use a new variable $\mathrm{W}$. a variable change is given by:

$$
T(x, t)=W(x, t) e^{\frac{v x}{2 \alpha}-\frac{v^{2} t}{4 \alpha}}
$$

After we make change all variables, the new governing equation is given by: 


$$
\begin{array}{r}
\frac{\partial^{2} W}{\partial \xi^{2}}+\frac{1}{k} g(\xi, t) \delta\left(\xi-P_{\xi}\right) e^{\frac{v \xi}{2 \alpha}-\frac{v^{2} t}{4 \alpha}} \\
=\frac{1}{\alpha} \frac{\partial W}{\partial t}
\end{array}
$$

And boundary conditions of first kind are given by:

$$
\begin{aligned}
& \mathrm{W}(0, \mathrm{t})=\mathrm{T}(0, \mathrm{t}) e^{\frac{v^{2} t}{4 \alpha}} \\
& \mathrm{W}(\mathrm{L}, \mathrm{t})=\mathrm{T}(\mathrm{L}, \mathrm{t}) e^{-\frac{v L}{2 \alpha}+\frac{v^{2} t}{4 \alpha}}
\end{aligned}
$$

Boundary condition of second and third kind:

$$
\begin{array}{r}
-k \frac{d T}{d \xi}+W(0, t)\left(\frac{-k v}{2 \alpha}+h_{\xi 1}\right) \\
=e^{\frac{v^{2} t}{4 \alpha}} h_{\xi 1} W_{\infty}+q_{\xi 1} \\
k \frac{d T}{d \xi}+W(L, t)\left(\frac{k v}{2 \alpha}+h_{\xi 2}\right) \\
=e^{\frac{v^{2} t}{4 \alpha}-\frac{v L}{2 \alpha}} h_{\xi 2} W_{\infty}+q_{\xi 2}
\end{array}
$$

And initial condition:

$$
\mathrm{W}(\xi, 0)=\mathrm{F}(\xi) e^{-\frac{v \xi}{2 \alpha}}
$$

Note that the boundary conditions of the second kind become to third kind, while boundary conditions of first and third kinds remain the same. We then determine the temperature distribution problem in calculating the variable $\mathrm{W}$ and then returning to the variable T using Eq. (8), the method of Green's functions for obtaining the analytical solution to this problem is used.

\section{NUMBERING SYSTEM}

Beck et al. (1992) use a numbering system for heat conduction problems according their boundary conditions. which may have six different combinations for this conditions, and thus, classify. The next table show this conditions.

Table 1. Types of boundary conditions.

\begin{tabular}{ccc}
\hline Notation & Name of boundary & Description \\
\hline 0 & Zeroth kind & No physical boundary \\
1 & Dirichlet & Prescrible temperature \\
2 & Neumann & Prescrible heat flux \\
3 & Robin & Convective condiction \\
4 & Fourth kind & Thin film, no convection \\
5 & Fifth kind & Thin film, convection \\
\hline
\end{tabular}

Therefore, for a boundary conditions problem the first and third kinds, we denote by X13, where X is problem direction, in $1 \mathrm{D}$ case problem.

The green's functions can be obtained in literature or calculated by some methods. here we use green's functions obtained in (Beck et al., 1992, p.584).

\section{PARTICULAR CASE}

Now we consider a heat conduction problem of first kind boundary condition in all boundaries, $x=0$ and $\mathrm{x}=\mathrm{L}, \mathrm{X} 11$ problem. This problem shows the method application, shown in previous section.

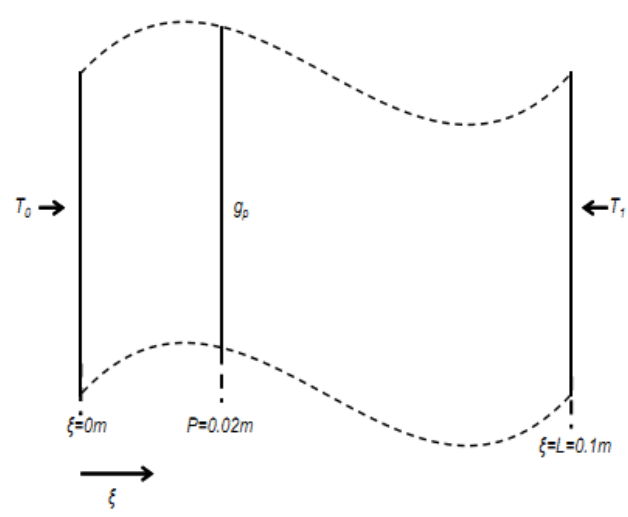

Figure 3. Problem.

The governing equation for this problem in $\mathrm{W}$ and $\xi$ variables is given by:

$$
\frac{\partial^{2} W}{\partial \xi^{2}}+\frac{1}{k} g(\xi, t) \delta\left(\xi-P_{\xi}\right) e^{\frac{v \xi}{2 \alpha}-\frac{v^{2} t}{4 \alpha}}=\frac{1}{\alpha} \frac{\partial W}{\partial t}
$$

Under boundary conditions:

$$
\begin{aligned}
& W(0, t)=0 \\
& W(L, t)=0
\end{aligned}
$$

And initial condition:

$$
\mathrm{W}(\xi, 0)=0
$$

Thus the Green's functions solution to the problem is given by:

$W(\xi, t)=$

$$
\frac{\alpha}{k} \int_{\tau=0}^{t} \int_{\xi^{\prime}=0}^{L} G_{11}\left(\xi, t \mid \xi^{\prime}, \tau\right) g\left(\xi^{\prime}, t\right) \delta\left(\xi^{\prime}-P_{\xi}\right) e^{\frac{v \xi^{\prime}}{2 \alpha}-\frac{v^{2} t}{4 \alpha}} d \xi^{\prime}
$$

The Green Function can be easily found in (Beck et al., 1992, p.584) and take the form: 


$$
\begin{aligned}
& G_{11}\left(\xi, t \mid \xi^{\prime}, \tau\right)= \\
& \quad \frac{2}{L} \sum_{m=1}^{\infty} e^{-\beta_{m}^{2} \alpha(t-\tau)} \sin \left(\beta_{m} \xi\right) \sin \left(\beta_{m} \xi^{\prime}\right)
\end{aligned}
$$

Where $\beta \mathrm{m}$ are eigenvalues and has the form:

$$
\beta_{m}=\frac{m \pi \xi}{L}
$$

Substituting Eq. (17) in Eq. (16), and using the property:

$$
\int_{-\infty}^{+\infty} f(x) \delta\left(x-x^{\prime}\right) d x^{\prime}=F\left(x^{\prime}\right)
$$

We have the problem solution after integrate with respect to $\xi$ '.

$W(\xi, t)$

$$
\begin{aligned}
& =\frac{2 \alpha g}{L k} \sum_{m=1}^{\infty} \sin \left(\beta_{m} \xi\right) \sin \left(\beta_{m} P_{\xi}\right) e^{\frac{\nu P_{\xi}}{2 \alpha}} \times
\end{aligned}
$$

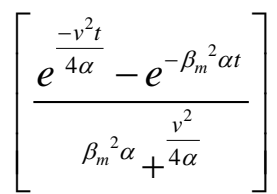

Therefore the solution is given by:

$$
T(\xi, t)=W(\xi, t) e^{\frac{v \xi}{2 \alpha}-\frac{v^{2} t}{4 \alpha}}
$$

The graph solution is show by Fig. (3) using:

$$
\begin{aligned}
& \xi=0.1 \mathrm{~m} \\
& v=0.001 \mathrm{~m} / \mathrm{s} \\
& k=80.2 \mathrm{~W} / \mathrm{mK} \\
& \alpha=23.1 \times 10^{-6} \mathrm{~m}^{2} / \mathrm{s} \\
& g(\xi, t)=1 \times 10^{5} \mathrm{~W} / \mathrm{m}^{3} \\
& P_{\xi}=0.02 \mathrm{~m}
\end{aligned}
$$

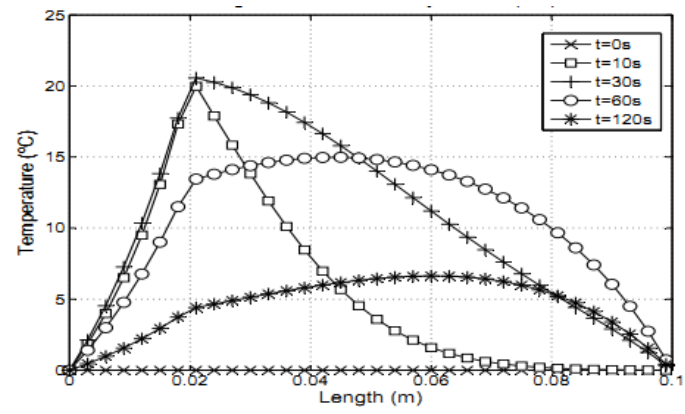

Figure 4. Temperature distribution for a moving solid.
We can observe the response of temperature due to heat generation, at a constant speed. Note the effect of temperature caused by the moving solid along the axis $\xi$ positive, also we can observe the response of temperature using the same solution but with zero speed, Fig. (4), in this case the moving problem $\xi 11$ reduces a fixed problem.

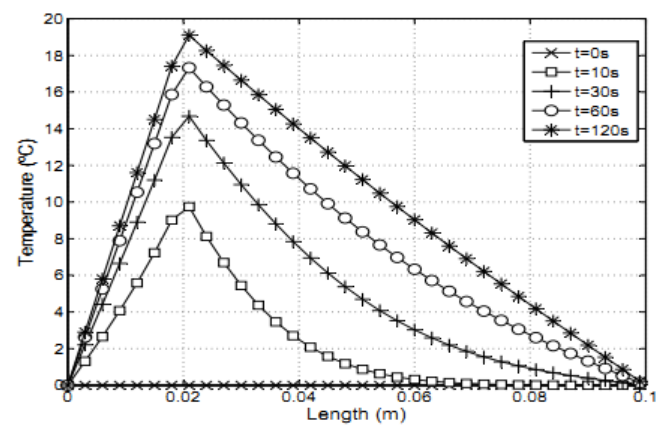

Figure 5. Temperature responses for fixed axis.

\section{VERIFICATION}

The analytic solution for problem $\xi 11$ was presented in previous section, can be compared with another solution, analytical or numerical. In order to have reliability and security for your use, in this section we will compare the solution obtained making $\mathrm{v} \rightarrow 0$ and compare with solution $\xi 11$ of fixed source easily obtained by Green function.

This solution has the form

$$
\begin{aligned}
& T(x, t)= \\
& \frac{2 \alpha g}{L k} \sum_{m=1}^{\infty} \sin \left(\beta_{m} x\right) \sin \left(\beta_{m} P_{x}\right)\left(\frac{1-e^{-\beta_{m}{ }^{2} \alpha t}}{\beta_{m}{ }^{2} \alpha}\right)
\end{aligned}
$$

Using the same thermo physical proprieties and times, next figure show the temperature field for this case.

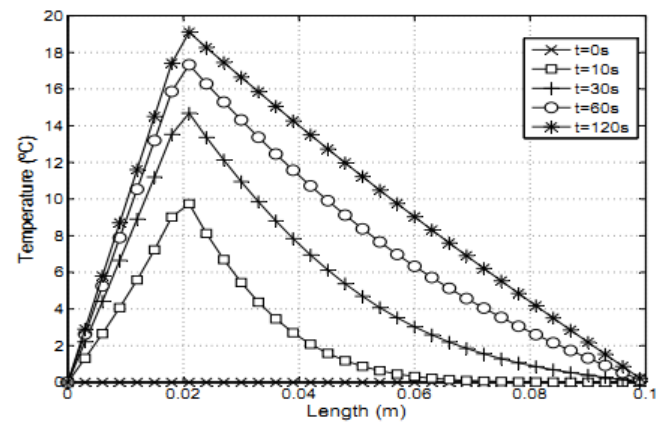

Figure 6. Temperature field for X11 fixed problem.

Now we consider the solution given by Eq. (19) and make $\mathrm{v} \rightarrow 0$ and comparison with solution shown in Eq. (21). The next figures show this effect. 


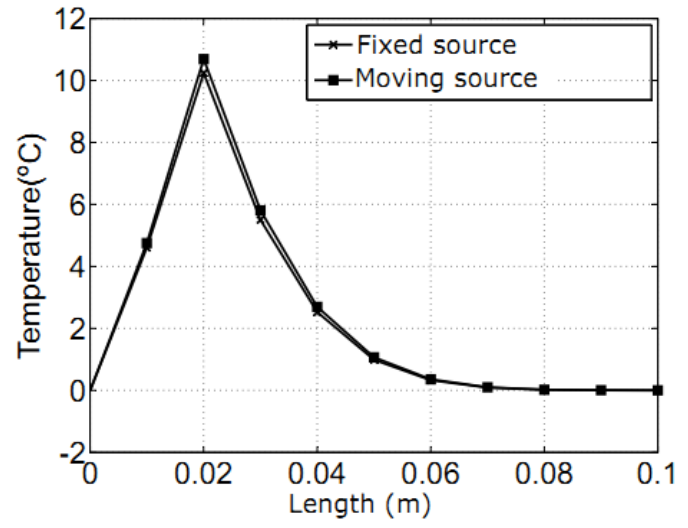

Figure 7. Comparison between fixed source and moving source for $\mathrm{v}=0.0001 \mathrm{~m} / \mathrm{s}$ and $\mathrm{t}=20 \mathrm{~s}$.

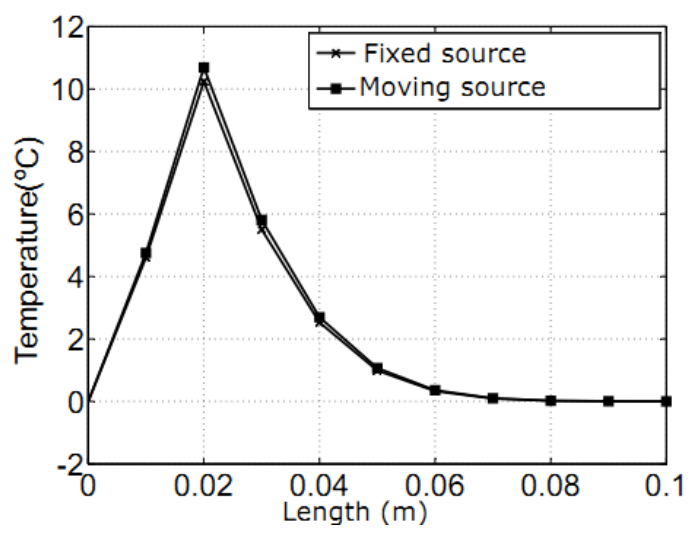

Figure 8. Comparison between fixed source and moving source for $\mathrm{v}=0.00005 \mathrm{~m} / \mathrm{s}$ and $\mathrm{t}=20 \mathrm{~s}$.

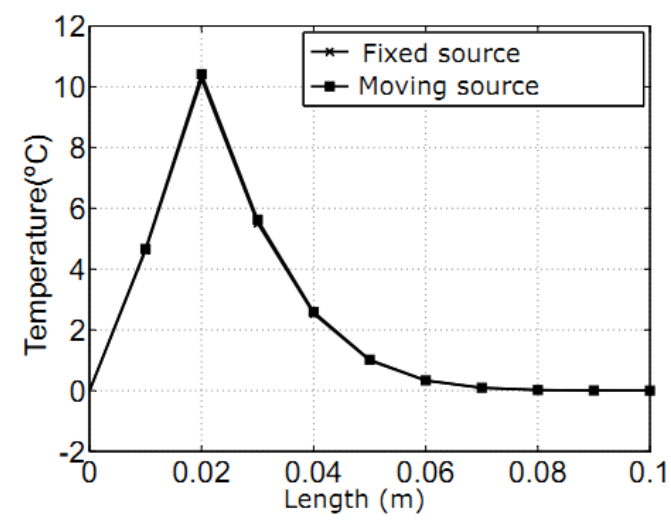

Figure 9. Comparison between fixed source and moving source for $\mathrm{v}=0.00002 \mathrm{~m} / \mathrm{s}$ and $\mathrm{t}=20 \mathrm{~s}$.

In this three figures previously shown, we can note that, the solution for moving source is the same solution for fixed case at small velocities, thus, we consider validated by this analysis.

Another possible comparison is by solutions obtained for numerical methods, remember that we can't say which validated an analytical solution by numerical method; just we make a comparison in this case. Next figure show this comparison.
So, we will simulate this problem with the boundary conditions in the commercial software COMSOL and compare with the results obtained analytically.

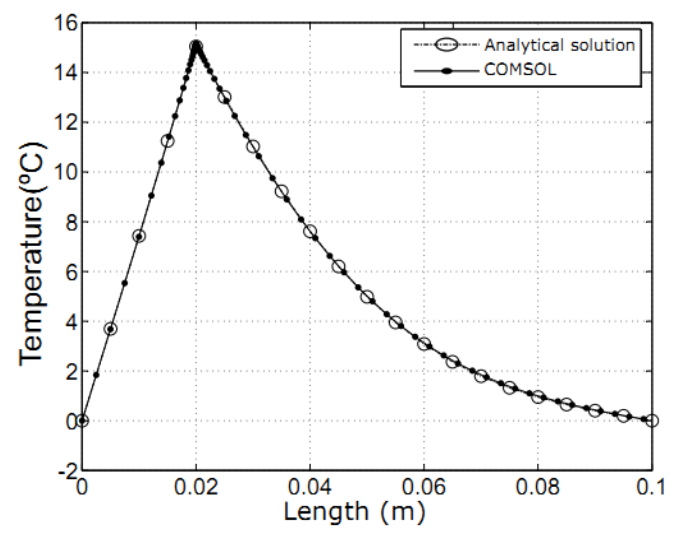

Figure 10. Comparison between analytical solution and numerical solution.

In the previously shown graph, we can note that two solutions, analytical and numerical, have the same behavior.

\section{CONCLUSIONS}

We can conclude that the use of analytical solutions in heat conduction is a important and strong tool for understanding of physical problems. even showing difficulty in understand the mathematical analysis used.

The method for calculate problems in various boundary conditions and 2D or 3D dimensions is the same shown, what is it advantage of use the Green's functions.

\section{ACKNOWLEDGEMENTS}

The author acknowledges the co-authors this work for their collaboration, and the funding agencies CAPES, FAPEMIG and CNPq.

\section{REFERENCES}

Araya, G., and Gutierrez, G., 2006, Analytical Solution for a Transient, Three-Dimensional Temperature Distribution due to a Moving Laser Beam, International Journal of Heat and Mass Transfer, Vol. 49, pp. 4124-4131.

Beck, J. V., Cole, K. D., Haji-Sheikh, A., and Litkouhi, B., 1992, Heat Conduction using Green's Function, Hemisphere Publishing Corporation.

Beck, J., and McMasters, R., 2004, Solutions for Multi-Dimensional Transient Heat Conduction with Solid Body Motion, International Journal of Heat and Mass Transfer, Vol. 47, pp. 3757-3768.

Fernandes, A. P, Sousa, P. F. B., Borges, V. L., and Guimaraes, G., 2010, Use of 3D-Transient 
Analytical Solution Based on Green's Function to Reduce Computational Time in Inverse Heat Conduction Problems, Journal of Applied Mathematical Modeling, Vol. 34, pp. 4040-4049.

Mohammad, H., Solaleh, S., Pouya B., and Sadaf, S., 2013, 3-D Transient Analytical Solution Based on Green's Function to Temperature Field in Friction Stir Welding, Journal of Applied Mathematical Modeling. Vol. 37, pp. 9685-9884.

Özisik, M. N., 1993, Heat Conduction, Wiley Interscience. 